\title{
Habitats: A Simple Way to Bridge Artifacts, Professions, and Theories in Ubiquitous Design
}

\author{
Martin Brynskov \\ Center for Interactive Spaces, University of Aarhus \\ Aabogade 34, DK-8200 Aarhus N \\ Denmark \\ brynskov@daimi.au.dk
}

\author{
Gunnar Kramp \\ Aarhus School of Architecture \\ Noerreport 20, DK-8000 Aarhus C \\ Denmark \\ gunnar.kramp@aarch.dk
}

\begin{abstract}
This paper briefly shows how product designers as well as information system designers may use the habitat framework as a tool to inform their understanding of the pervasive computing systems they are designing. This is done by (1) introducing the basic elements of habitats, (2) analyzing and comparing two empirical case-studies, one about life and death (emergency response at major incidents) and one about playfulness (children's pervasive play and gaming), and (3) discussing the usefulness of using habitats. The result is a number of real-world examples where we argue that using habitats as a simple common ground seems to be useful for professionals coming from quite different traditions.
\end{abstract}

\section{Categories and Subject Descriptors}

H.1.2 [Models and Principles]: User/Machine Systems human factors, human information processing.

\section{General Terms}

Design, Human Factors, Theory.

\section{Keywords}

Design support, design principles, interdisciplinary work, pervasive healthcare, pervasive play and gaming

\section{INTRODUCTION}

Our interest in this paper is to demonstrate some shared characteristics between work and play involving wireless networked devices. The reason for doing so is partly because designing pervasive technologies is still a field that is poorly understood, partly because we want to explore theories and methodspreferably simple ones - that can be shared among very diverse professional backgrounds, in this case industrial design and computer science/human-computer interaction.

The motive for sharing our experiences using habitats grew out of frustration while working in multidisciplinary teams that lacked a shared set of concepts and vocabulary to set the stage for design discussions about ubiquitous systems. Wishing to move beyond ad hoc, emergent, team-specific idiosyncrasies, we retrospectively used habitats to reformulate two design cases, one about rescue work and one about pervasive playfulness.

\section{HABITATS: THREE PERSPECTIVES}

The notion of habitats originally comes from biology as a definition of the relationship between the population of a specific species and a specific environment. The basic idea is that a habitat supports its inhabitants.

Wireless networked technology is an expander of our natural habitat. Hot spots in cities for accessing the internet is an obvious example of a habitat which supports its inhabitants, and where the relationship between its users and the environment is inseparable.

The expanded environment has thus given the need for clarification resulting for example in discussions regarding space and place (cf. Harrison and Dourish [6]). However space and place discussions do not take into consideration the root of the discussion as it relates to the world as we understand it today; as a configuration of places in a surrounding space. This understanding is related to our everyday physical world where places can be located by coordinates and space can be configured in 3D coordinate systems. By discussing space and place we constrain ourselves to the physical world of space and time in physics. Accordingly, habitats provide a much richer approach to describing, analyzing, and designing for wireless technology and leaps over the dichotomy of space-place discussions and into the fundamental relationship between ourselves and the our environment.

Our inspirational source is work done by May and Kristensen [9] where they introduce the notion of "Habitats for the Digitally Pervasive World". May and Kristensen conceive habitats as something that specifies some kind of locality, they comprise inhabitants and they provide support to their inhabitants. They identify three types of habitats: the physical, the informational, and the conceptual habitat. Others have developed the habitat concept further into a more consistent framework to support analysis and design of ubicomp systems $[3,1]$. The fundamental feature of the habitat framework is that it offers three perspectives on a habitat in which activities take place. While the previous literature on (digital) habitats has been confusing the 'habitat' as a whole with the three perspectives, we refer to habitats only to denote the compound concept (except when quoting others).

- A habitat is a chunk of space-time that is designed or has evolved to support a delimited set of activities by offering physical artifacts and information sources useful for conducting the activities.

From this definition, it follows that a habitat should be characterized along three dimensions. These three dimensions of a habitat are called perspectives or parts:

Bunskov \& Gunnar Kramp, 2007

Published by the British Computer Society

Volume 2 Proceedings of the 21st BCS HCI Group Conference

HCI 2007, 3-7 September 2007, Lancaster University, UK Devina Ramduny-Ellis \& Dorothy Rachovides (Editors)
- The physical perspective: the physical boundaries and qualities of the habitat including artifacts.

- The informational perspective: the signs (digital and nondigital) available to participants (access and reference area). 
- The pragmatic perspective: the action affordances offered by the habitat and the role-requirements.

Habitats are structured around activities. Activities consist of actions subsumed under a shared goal, and participants play roles in relation to actions and activities.

\subsection{Physical Perspective}

The physical aspect is what Gibson [5] defines as medium, substances, and surfaces. While Gibson's description is very concrete, another-and in our view more helpful — definition is: the part of the habitat where the activities of the inhabitants take place, i.e. the physical perspective. Physical parts of a habitat can thus be, e.g., a car, a farmers field, an office, and physical parts can be nested in each other. For example an office can be nested in a building, a building can be nested in at town and so forth, providing physical scale.

\subsection{Informational Perspective}

A hotspot in a city is part of a habitat. The range of the wireless network may define the extent of the habitat. However, while the technology defines the extent of the habitat, there is also a qualitative aspect, namely the information which is conveyed in the habitat. May and Kristensen give an example of an email system as an "informational habitat." But while May and Kristensen see the email system as a habitat in itself, we follow the definition above and see the hotspot through different perspectives. Thus the hotspot may been seen both from the physical perspective and the informational perspective.

In the informational perspective, Brynskov and Andersen [3] distinguishes between the access area and the reference area. The access area is the area where the inhabitants have access to the information, while the reference area is the object of the information. The access area and the reference area are connected through a medium. For example, the hotspot area is the access area and the internet is the reference area which is connected through a medium such as a laptop.

\subsection{Pragmatic Perspective}

Brynskov and Andersen [3] have a slightly different approach than May and Kristensen as they instead of "conceptual habitats" use the notion of "pragmatic habitats," which are characterized by being a "shared mental space" which sets the boundaries for the inhabitants behavior. In practice this means that there are habitats where skills, knowledge, obligations and rights of the inhabitants are defined implicitly or explicitly, and which are necessary to possess or comply with in order to be a inhabitant in the habitat. Brynskov and Andersen argue that the pragmatic perspective cannot be separated from intentions and expectations, thus resulting in what they call potential activity and realized activity. It follows that the pragmatic perspective can consist of potential activities defined as roles, while the realized activity is the potential activity realized by a person or digital agent, thus becoming the filler of the role. This relation between potential and realized activity becomes important, as can be seen in the later examples, because the informational perspective often will facilitate either a remote filler of a realized activity and/or facilitate that several users can join efforts to fill one role.

\section{A COMMON STAGE}

In the following we present two simple uses of the habitat framework in creating a foundation for staging the activities taking place in the world of wireless networked technology. While others have developed the framework in more depth and detail, we only apply it to our design cases to see what can be gained from thinking in terms of habitats. The link between the two cases is only that they are faced with somewhat similar problems as a consequence of designing ubicomp systems.

Activities involving wireless networked technologies often increase complexity due to multiple actors, physical and digital constraints, and the distributed use of artifacts. An activity taking place in one situation can have a profound impact on another situation in another setting. This complexity is furthermore worsened by the number of professions and theories involved in the design process.

The habitat framework presented here is not to be interpreted as a complete formula for making successful design solutions in the complex domain of ubiquitous computing. It should rather be understood as a model for distilling the activities taking place at different levels, in order to provide simplicity for design, while still retaining important distinctions that often characterize ubiquitous computing systems. As a consequence, habitats need to be complemented by other methods and disciplines as we shall see. The framework merely offers a common stage to be populated by a range of artifacts, professions, and theories.

\subsection{Habitat Analyses of Two Case Examples}

We provide a brief analysis of illustrative examples from two cases: (A) a prototype of the "biomonitor" [7] which is used at major incident sites to provide vital information about wounded victims, and (B) a prototype of a pervasive game called DARE! [4], in which children can use mobile phones and pervasive technologies to compose and send challenges to each other, e.g. to take a picture of something. These experiences with habitat analyses of the biomonitor and the game are described from the perspectives of an industrial designer and an HCI person respectively.

A habitat can be defined as a place which supports the activities of its inhabitants, and the inhabitants relationship to the habitat can be defined as one or several roles. When a major incident occurs, e.g. a railroad accident or an earthquake, the place where it happens is not a habitat for the victims. On the contrary, it is significant that the reason for the accident will be because something in the environment has not supported the activity taking place. For example that a railway does not support two trains running towards the same point on one track.

What needs to be done for the rescue personnel at the arrival is to try to force the incident site into a state where the habitat supports the rescue activities taking place. This transformation is not trivial. The physical environment is non-predictable, the different communities of practice seldom work together under these conditions, and the people in charge may never have met in person before. Furthermore, communication between personnel, coordination of resources etc. is complex and, finally, it all takes places with an overhanging time pressure. There seems to be a general acceptance of the term "the golden hour" [8], which implies that chances of recovering from a serious trauma are significantly better if the patient is treated within an hour from the time of the accident.

Similarly, when a group of children engaged in the DARE! game move between locations, the interaction potential changes. DARE! is a simple social activity that consists of three phases: composition, performance, and evaluation of a dare. The motive behind this kind of playfulness is to provide a pervasive platform which supports humor, friendly conflict, and identity construction. In some places the children have time and opportunity to challenge each other and to take interesting pictures, while in other situations it is less desirable or possible.

A way of trying to get an overview of people, artifacts, and communication technologies is to utilize the notion of habitats. We have in the example below (Figure 1) classified the organi- 
A) BIOMONITOR PROTOTYPE

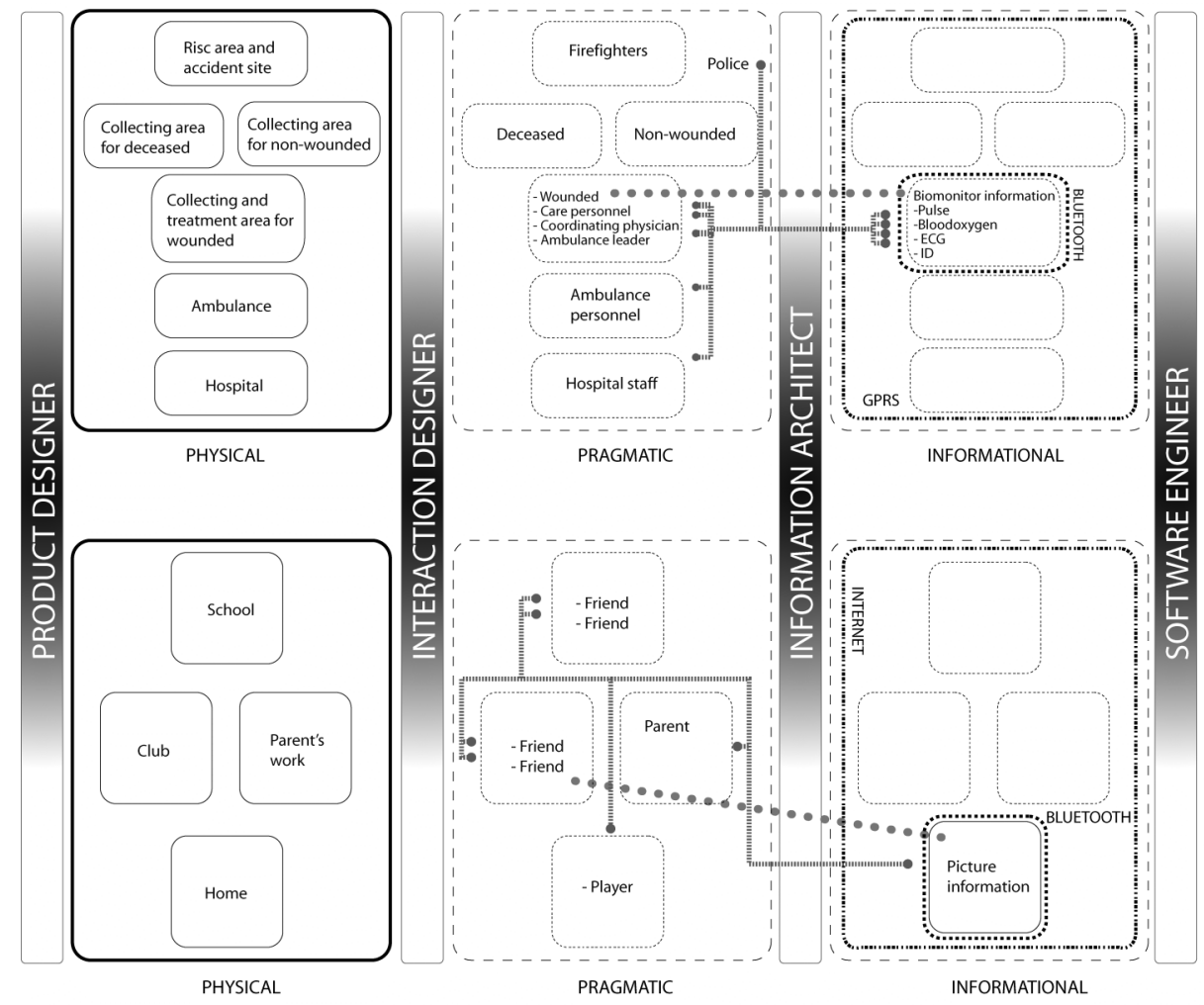

mistribution of data

Connection between source and data

B) DARE PROTOTYPE

Figure 1. Habitat analyses of two cases: (A) the biomonitor prototype and (B) the DARE! game prototype.

zation of both the major incident case and the pervasive play case in the three perspectives: the physical, the informational, and the pragmatic perspective in order to provide an overview of the use of biomonitors on an incident site and of a simple picture dare. The four vertical bars, illustrate the "positioning" of product design, interaction design, information architecture, and software engineering in relation to the three perspectives. Evidently, these positions are in reality much more intertwined and complex, but we use the illustration to show how the three perspectives on the habitat can encompass the involved professions.

\subsubsection{Physical perspective}

If we compare the play scenario to the emergency response, we see a similarity in the fact that none of designers (general rescue operations procedure planners and dare composers) know exactly what situation their designed activity will play out in. In fact, they can count on the unexpected to play a major role. The difference is, however, that the emergency response activity has a much clearer procedure that ties the rules and roles to physical space. The participants also know that everyone (at least the professionals) will do their best to adhere to the plan. Dares, on the other hand, involve a constant element of surprise and competition. There are dares that are classics, but overall, even they are just a few stable elements in an ever-changing chain of exchanges. On a general level, dares may or may not be tied to designated parts of physical space.

The consequences of this on design is that both systems should support adaptation to the physical surroundings. But there are differences. The emergence response system should pay close attention to the ideal that is laid out in the detailed procedures in order that safety and accountability is given first priority. The game, on the other hand, should highlight opportunities for fun and social exchange. The first part of a habitat analysis highlights the physical properties of the habitat and show how people, artifacts, and places are related to each other. The actual appearance may be naturalistic, e.g. projected onto a real map, or abstract, focusing only on relationships and accessibility. The two examples in Figure 1 are abstract. In the case of the biomonitor, they show how the different zones of the incident site are separated, and in the play scenario they show the different places that kids visit throughout a regular school day.

\subsubsection{Informational perspective}

The informational aspects of the dare activities consist of the digital information in the system as well as of whatever information that may be left in the physical environment. Such nondigital or analog information includes physical markers of tags, bystanders informing or helping the performer, and simple signs, e.g. "Camera phones not allowed". Simply the fact the we force ourselves to consider how information that is not modeled in the system at all may play a role in the activities is important. It opens up the space for thinking creatively both at system design time and subsequently at dare design time.

An analysis of the informational habitat also visualizes the relationships between where information is actually accessible (the access areas, e.g. a screen or a tag) and what it refers to (the reference area, e.g. a person or a place). By mapping these areas we get a sense of where what is available. Again this may lead to a larger, more relevant decision space at both system design time and dare design time, potentially making way for better solutions.

The system should support reconfigurations of informational parts of habitats by allowing the participants to change and 
combine access and reference areas. By modeling this relationship in a habitat, the designers can explore and analyze issues that are problematic and need special attention or discover opportunities to provide flexible activity support.

In DARE!, one such situation may occur if the recipient of a challenge sees an opportunity to fulfill the requirements by enrolling proxies, e.g. a friend that is present somewhere close to an object that must be found or photographed. This could also be described as "realizing an activity by combining inhabitants to fill a role."

The other example shows what information that is made available by the biomonitor within the area for collecting and treating the wounded. It also shows in which other areas this information might potentially be useful, e.g. if a doctor is not physically present at the site but sits back in the hospital.

Again there are similarities and differences between the two cases. Both the emergency response work and DARE! will benefit from providing explicit support for altering the informational habitat in order to establish a situation that is desirable for some reason (e.g. using audio and video feeds to facilitate the joint effort of ambulance personnel and a hospital physician in a case where they cannot be physically co-located). The differences are mostly concerning of reliability and quality of service, but if a particular workaround is the only solution in a critical situation, it should certainly not be disregarded.

\subsubsection{Pragmatic Perspective}

The pragmatic aspects of habitats are characterized by the roles that are given and created. One interesting feature by looking at the play scenario is that it does not necessarily matter whether a role is played by a human being or an agent, e.g. a robot or a piece of software. One can easily imagine challenges where the clever recipient can enroll non-human agents in his or her efforts to meet the goal of the challenge.

This is not all that different from the emergency response situation, except emergency response is a mission-critical activity. People may die, if something goes wrong and a wrong decision is made. As a consequence, only roles that are more or less trivial may be filled by an autonomous agent-unless, maybe, that using an agent is the only way out. Then the desperate situation may warrant the user's enrollment of an autonomous agent. Such levels of control are well-known from the transport sector, e.g. airplanes and ships that change between fully automated, semi-automatic, and manual maneuvering [2].

Describing and mapping the pragmatic perspective of a habitat may very well draw on thick descriptions that are distilled into simpler models of the social configurations. These may be related to the physical perspective, as mentioned earlier. But they may also be considered in connection to the informational perspective. Figure 1 shows the pragmatic aspects of the two habitats as the distribution of roles in the physical space. This example does not distinguish between potential and realized activities.

\section{CONCLUSION}

In this paper, we have turned the attention towards the notion of habitats not only as a physical environment supporting its inhabitants, but also as an environment consisting of informational and pragmatic aspects. All three perspectives are related to our perceived world and the human scale centered around our activities. However, the existence of and relationships between the perspectives are not well recognized in current theories and methods as we still lack sufficient transparency across the domains of physical and digital design theories and methods to include this in our solutions as users and designers. Nevertheless, we have used the notion of habitats as a foundation for framing activities in wireless networked environments in general and our two use cases specifically.

While initially frustrated, we have found the framework helpful in analyzing our work with pervasive and palpable computing. The set of concepts seem simple enough to be shared easily. The examples that we have highlighted above especially showed how it can be used to expose emergent use situations during design. However, habitats do not remove the need for more specific models, theories, and professional skills. Furthermore, although we have both worked separately on different projects, and one participant has never used the framework before, this is only a weak validation of the efficacy of using habitats. Just because our frustration was eased there is no guarantee that others will perceive habitats as helpful in ubiquitous design work.

It is our conclusion that even though the framework can be very simple, or maybe because of this, the subdivision of habitats into three perspectives-physical, informational, and pragmatic - can provide a helpful foundation for framing the stage of ubiquitous, pervasive, and palpable computing across disciplines. It is also our conclusion that more work is needed to mature the framework towards sustaining the balance of simplicity and power of expression as complexity of the design domain increases.

\section{ACKNOWLEDGMENTS}

We wish to thank our colleagues who worked with us on the two projects. We would also like to thank the anonymous reviewers for helpful comments. This work was supported by the Center for Interactive Spaces at the University of Aarhus (ISIS Katrinebjerg project \#122) and the Palpable Computing EU project, PalCom (IST 002057).

\section{REFERENCES}

[1] Andersen, P.B. and Brynskov, M. The semiotics of smart appliances and pervasive computing. In: Gudwin, R. and Queiroz, J. (eds.) Semiotics and Intelligent Systems Development, Idea Group, Hershey, NJ, 2006, 211-255.

[2] Bødker, S. and Andersen, P.B. Complex Mediation. Human-Computer Interaction, 20 (4), 2005, 353-402.

[3] Brynskov, M. and Andersen, P.B. Habitats, Activities, and Signs. In Proc. of Organisational Semiotics 2004, INSTICC Press, 128-151.

[4] Brynskov, M. and Ludvigsen, M. Mock Games: A New Genre of Pervasive Play. In Proc. of DIS 2006, ACM Press.

[5] Gibson, J.J. The Ecological Approach to Visual Perception. Houghton Mifflin, Boston, 1979.

[6] Harrison, S. and Dourish, P. Re-place-ing space: The roles of space and place in collaborative systems. In Proc. of CSCW 1996, ACM Press, 67-76.

[7] Kramp, G., Kristensen, M., and Pedersen, J.F. Physical and digital design of the BlueBio biomonitoring system prototype, to be used in emergency medical response. In Proc. of Pervasive Healthcare 2006.

[8] Lerner, E.B. and Moscati, R.M. The Golden Hour: Scientific Fact or Medical "Urban Legend"? Academic Emergency Medicine, 8 (7), 2001, 758-760.

[9] May, D. and Kristensen, B.B. Habitats for the Digitally Pervasive World. In: Andersen, P.B. and Qvortrup, L. (eds.) Virtual Applications: Applications with Virtual Inhabited 3D Worlds, Springer, London, 2004. 